\title{
NONCONTACT TESTING \\ OF UNDERGROUND PIPELINES CORROSION
}

\author{
Roman Dzhala ${ }^{1}$ \\ Vasyl Dzhala ${ }^{2}$ \\ Bohdan Verbenets'3
}

DOI: https://doi.org/10.30525/978-9934-588-53-2-53

\begin{abstract}
Underground pipelines (UP) transport gas, oil, water, raw materials and products of chemical industry. Influence of environment can lead to corrosion damages of pipelines. It causes large losses and troubles with supplies of transported products, pollution of environment, accidents and catastrophes.

For increase of reliability and continuation of exploitation time of metallic pipelines complex corrosion protection (CP) is used. Insulation and cathodic polarization are used. To prevent of irreversible corrosion damages is necessary to establish $\mathrm{CP}$ on time and in appropriate places. It requires periodic surveys for detection of corrosion hazardous places. Traditional contact electromagnetic methods of UP inspection from the ground surface are labor-consuming and not informative enough. In-tube inspection can detect existing damages of pipeline metal but cannot obtain needed information about state of anti-corrosion protection. Efficiency of surveys significantly increases with use of contactless methods, in particular, non-contact current measurement (CM). But for their broad use appropriate tools for selection and data processing are needed.
\end{abstract}

\footnotetext{
${ }^{1}$ Doctor of Technical Sciences,

Head of the Laboratory of Electrophysical Methods of Non-Destructive Testing,

H.V. Karpenko Physico-Mechanical Institute,

National Academy of Sciences of Ukraine, Ukraine

${ }^{2}$ Candidate of Technical Sciences, Senior Researcher at the Laboratory

of Electrophysical Methods of Non-Destructive Testing,

H.V. Karpenko Physico-Mechanical Institute,

National Academy of Sciences of Ukraine

${ }^{3}$ Candidate of Technical Sciences, Senior Researcher at the Laboratory

of Electrophysical Methods of Non-Destructive Testing,

H.V. Karpenko Physico-Mechanical Institute,

National Academy of Sciences of Ukraine
} 
Subject of this study is contactless method for UP current measuring, control of insulation condition parameters and detection of places of corrosion. Methodology of study is based on triune mathematical model of UP electromagnetic field. It consists of solutions of electrodynamic boundary value problems, theory of electric circuit with distributed parameters and calculation of electric and magnetic fields of spreading currents in conducting medium. The aim of the study is development of contactless method and creation of technical devices for quality estimation of insulation for express surveys and control of $\mathrm{CP}$ state parameters along the route.

As consequence of work in Karpenko Physico-Mechanical Institute of Natinal Academy of Sciences of Ukraine method of contactless current measurement is developed and new CM devices for control of UP CP state are created. New technology of integral, differential and local inspection and quantitative estimation of UP CP parameters are developed. New methods for express detection of places with unsatisfying insulation, determination of cathodic protective current density distribution, transitional resistance "pipe-to-ground" and its components (insulation, ground and polarization resistances) is proposed. Efficiency of created method and apparatus are tested by comparison with results of traditional contact measurements on test benches and during surveys on UP in operation. Designed method and $\mathrm{CM}$ apparatus significantly increase effectiveness of diagnostic inspections, quantity and quality of received information about state of UP CP.

\section{Introduction}

Underground pipelines (UP) play important role in industry, everyday life and are important strategic factor of economic complex. In different regions of the world there are more the 2 million $\mathrm{km}$ of pipelines, through which gas, oil, raw materials and products of chemical industry are transported.

Influence of environment can lead to corrosion damages of pipelines and other metallic constructions [1-3]. It causes large losses and troubles with supplies of transported products, pollution of environment, accidents and catastrophes. Corrosion accident rate of UP is $27 \%$ and increases to unsuitability of construction for further operation. For increase of reliability and continuation of operation time of metallic pipelines complex corrosion protection $(\mathrm{CP})$ is used. For this purpose insulation and cathodic polarization 
are used (electrochemical CP). To prevent of irreversible corrosion damages is necessary to establish $\mathrm{CP}$ on time and in appropriate places. It requires periodic surveys for detection of corrosion hazardous places. Scientists of different specialties are working to solve this complex problem.

Traditionally UP are investigated using contact electrometric methods from the ground $[1 ; 2 ; 4]$. This is labor-consuming and not informative enough. In-pipe pigging inspection can detect existing damages of pipeline metal, but cannot obtain needed information about state of CP. Efficiency of testing can be significantly increased using non-contact methods of measurement. But for their broad use appropriate methods and tools for data selection and its processing for qualitative estimation of control object parameters, which is actual problem.

In Karpenko Physico-Mechanical Institute of the National Academy of Sciences of Ukraine method of non-contact CM is developed and new CM devices for control of UP CP state are created. New technology of integral, differential and local inspection and quantitative estimation of CP parameters distribution along route are developed. The goal of the study is development of non-contact CM method and creation of technical tools for detection of insulation and cathodic polarization for express testing and control of CP state of metallic UP. For achieving of this goal next scientific problems must be solved:

- To analyze current state of methods for corrosion control of UP and informative signs of electromagnetic field on UP route; to explore current leakage effect in UP;

- To develop method and to create apparatus for non-contact CM, placement, orientation and depth of occurrence determination;

- To develop method for determination of cathodic protection current distribution, transitional resistance and express control of CP parameters on UP.

Methodology of study is based on triune mathematical model of UP electromagnetic field. It consists of solutions of electrodynamic boundary value problems, theory of electric circuits with distributed parameters and calculation of electric and magnetic fields of spreading currents in conducting medium. Investigation and equipment development are based on theory of informational-measurement technology, mathematical simulation with use of empirical and heuristic approaches, laboratory and 
full-scale testing. Efficiency of created method and equipment are tested by comparison with results of traditional contact measurements on test benches and during surveys on UP in operation.

In this article analysis of current state of UP corrosion control system, results of scientific and technical development of methods and tools for diagnostic surveys, UP CP state control and some of their applications are showed.

\section{Analysis of evolution of UP corrosion control systems}

As result of many investigation and practice of long operation complex method of CP for steel underground and underwater pipelines (UP) using insulation and cathodic polarization are developed [1-4]. During building and operation under influence of aggressive environment insulation are decayed, aged, usually faster then metal of UP. Electrodynamic situation along route changes over time. It requires periodic testing of $\mathrm{CP}$ state to make reasonable planning of timely repair for prevention of damages and accidents.

Today problem of diagnostic testing of metallic UP state is connected to end of service life of significant number of arterial gas and oil pipelines, increase of number of damages, accidents, catastrophes. Investigation of corrosion state, estimation of reliability for further operation, determination of types and scope of repairs for providing reliable functioning of pipelines are needed [4]. This problem causes special attention because many potentially dangerous arterial pipelines of high pressures are laid on densely populated territory. Problem is complicated because of large length of pipelines, existing of obstacles and inhomogeneity of conditions along route. Inhomogeneity of environment characteristics causes irregularity of corrosion process in different places on route. Thus timely detection of most probable places of corrosion is very important.

Information-measuring systems (IMS) are used for investigation, control, diagnostic and monitoring of UP technical state. These systems can be divided on three types [4] (Figure 1). Stationary IMS are implemented using systems of sensors placed in certain points on route and connected to control point. Such systems provide only information obtained in these points and integrally between them. In-pipe pigging inspection of metallic pipeline walls is implemented with special magnetic or ultra-sonic systems 
$[5 ; 6]$, which are moving through pipeline without stopping the flow of product. However, in-pipe inspection can detect existing damages of pipeline metal but cannot obtain needed information about state of $\mathrm{CP}$. Therefore, investigations of UP outer surface state (insulation, electric polarization) and environment are needed. Systems of the field expeditionary investigation provide most information about $\mathrm{CP}$ state. Three types of IMS can be distinguished among them (Figure 1).

Traditionally contact electrometric methods are used for CP inspection $[1 ; 2 ; 4]$. They are simple enough both in idea and implementation. However, their efficiency is limited by labor intensity and difficulties with providing enough reliable contact of measuring electrodes with pipeline and ground. Besides, in electrometric methods only part of electric field information is used. Magnetic field was more often used to placement determination of pipelines.

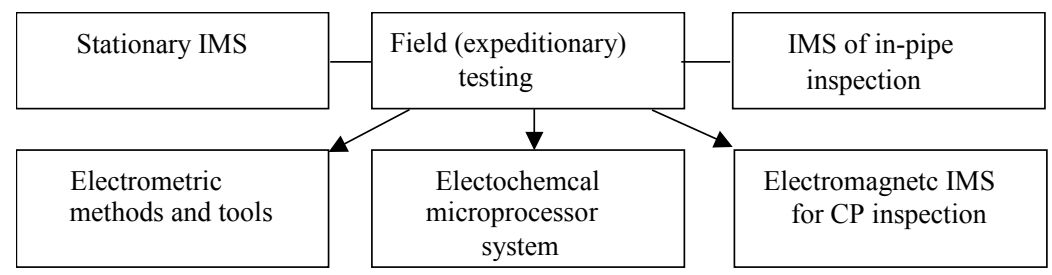

Figure 1. Systems of testing main pipelines

Non-contact methods of inspection have significant advantages in comparison with traditional contact methods due to mobility, efficiency and amount of information [4;6-8]. But they need special measuring tools, thus, they hadn't such broad use. For implementation of non-contact methods complex investigation of informative signs of field and measured signals, creation of algorithms, measuring tools and data processing were necessary.

Methods for inspection of object without direct access, has been developed in solving problems in field theory, measurement, data processing in the field of physicometrics, geophysics, and also in problems of inspection of hidden objects and conductive engineering communications, nondestructive testing and technical diagnostics [6;8]. Problem of selection measured signals, quantitative estimation of their informative signs and determination due to them of object parameters is one of the most important 
among problems, connected to creation of high-efficient IMS for search and inspection of hidden objects [8]. This complex problem acquired special relevance for problems of increasing efficiency and informativeness of UP $\mathrm{CP}$ inspection and determination of most probable place of corrosion on arterial pipelines.

Solution of this problem is connected first of all to study of relationships between spatial and frequency characteristics of electromagnetic (EM) fields and object parameters and selection of measured field characteristics considering technical possibilities of measurement implementation, creation of electromagnetic IMS, its algorithmic, hardware, software and methodic providing [8; 9]. Thus relevance of research works caused by necessity of investigation of UP EM field informative signs, development of new methods of non-contact CM and determination of object parameters, creation of scientific and methodic foundations for construction and functioning of electromagnetic IMS with appropriate algrythmic, methodic and technical equipment, designed for inspection of UP and associated with them metallic constructions.

Currents have valuable information about corrosion processes of metal in conductive medium. Current distribution is most sensitive to composition of environment and insulation state. But use of traditional contact method for $\mathrm{CM}$ in UP require special arrangement and thus is unusable for express testing $[4 ; 6-8]$.

For increasing of efficiency and informativeness of corrosion state control in arterial pipelines researches and development of electromagnetic method and tools of UP diagnostic testing was conducted in Karpenko Physico-Mechanical Institute of the National Academy of Sciences of Ukraine. Development of these method of non-contact UP inspection is connected to research works of PMI about problems of informative signs detection for medium, construction materials, orientational and irradiating structures, and also electromagnetic measurements, IMS, non-destructive testing, technical diagnostic and corrosion researches. Aspects of these problems were also developed in other institues of the NASU, in institutes and on enterprises of Naftogaz of Ukraine, and in Europian, American and Asian countries [4;6-10].

Currently non-contact methods have broad use only for pipelines and cables placement determination, sometimes for detection of insulation damages 
$[4 ; 7 ; 8]$. Possibilities of non-contact CM for control of current distribution in cathodic protection stations, and for quantitative estimation of distribution of UP transitive resistance and its components are little used. Therefore, popularization, broad use and further development of these method are relevant.

\section{Theoretical foundations of UP non-contact testing}

Based on previous development of electromagnetic field theory for cylindrical partially shielded channeling and radiating structure triune mathematical model (TUMM) of EM field of steel insulated underground pipeline is proposed [8]. This model is consists of solutions of electrodynamic boundary value problems, theory of electric circuits with distributed parameters and calculation of electric and magnetic fields of spreading currents in conducting medium (Figure 2).

From electrodynamic point of view steel insulated UP (Figure 3) is cylindrical multilayer structure. Electrical and magnetical components of its field can be described using longitudinal components of electric and magnetic Hertz vector, that satisfy Helmholtz equation, to which Maxwell equations comes down. Field changing with frequency $\omega$ in each of structure subregions is represented by decomposition on elementary wave functions like

$$
\Pi_{z l}^{e, m}(r, \phi, z, \omega)=\int \sum_{n} R_{n}^{e, m}\left(v_{l} r\right) \cdot \Phi_{n}^{e, m}(\phi) \exp ( \pm i \gamma z) \exp (i \omega t) d \gamma,
$$

where $v_{l}^{2}=k_{l}^{2}-\gamma^{2}$ is transversal wave numbers of subregions $(l=b, T, i, c)$; $k_{l}=\sqrt{\omega^{2} \varepsilon_{l} \mu_{l}-i \omega \mu_{l} \sigma_{l}}$ is wave number of medium; $a_{l}$ is radius of pipeline and insulation; radial field functions $R_{n}^{e, m}\left(v_{l} r\right)$ are combinations of Bessel functions due to boundary values and describe electromagnetic and metric parameters of structure; constants of propagation $\gamma$ can be established from dispersion transcendent equation obtained from boundary value systems [6; 8].

Field spreads along pipeline, in general, like quasi-TEM-wave, which exists at all (including zero) frequencies $[6 ; 8 ; 9]$. Therefore, we can represent UP as long line, which distributed parameters $R, L, C, G$ is determined by solving of electrodynamic boundary problems. Dependencies of field spreading on structure parameters are explored using equivalent circuits, which visually show electromagnetic processes and facilitate its analysis. For natural soils, which usually is non-magnetic and weakly conductive for frequencies less than $1500 \mathrm{~Hz}$, UP current magentic field (1) at the small distances from pipeline is described by formulae 


\section{Electrodynamic boundary problems}

- Structure parameters.

$\sqrt{ }$ Characteristics, informative signs of pipeline EM field

$$
\vec{E}, \vec{H}(r, \varphi, z ; a, \sigma, \varepsilon, \mu ; \vec{j}, \omega)
$$

\begin{tabular}{|c|c|c|}
\hline $\begin{array}{l}\text { Integral parameters } \\
\quad R, L, C, G \\
\text { of equivalent circuit }\end{array}$ & & $\begin{array}{l}\text { abstantiation of model, } \\
\text { limits of use }\end{array}$ \\
\hline $\begin{array}{c}\text { Circuits with distributed } \\
\text { parameters }\end{array}$ & \multirow{2}{*}{$\begin{array}{c}\text { Selection of } \\
\text { measurements; } \\
\text { pipeline inspection } \\
\text { algorithms }\end{array}$} & Current magnetic field \\
\hline $\begin{array}{l}- \text { Pipeline. } \\
\sqrt{ } \text { Voltage and current } \\
\text { distribution; } \\
\sqrt{ } \text { attenuation of field } \\
\text { along the pipeline. } \\
\quad J, V, \gamma(R, C, L, \omega)\end{array}$ & & $\begin{array}{l}\text { - Currents in pipeline } \\
\text { and ground. } \\
\sqrt{ } \text { Informative signs of } \\
\text { current magnetic field } \\
\text { distribution } \\
\quad \vec{H}(x, y, z ; \vec{j})\end{array}$ \\
\hline
\end{tabular}

Figure 2. Structure of triune mathematical model (TUMM) of UP EM field

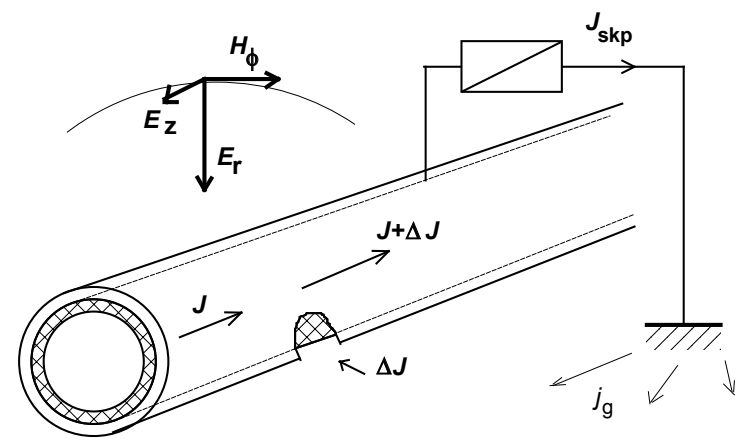

Figure 3. Underground pipeline with insulation and station of cathodic protection (SCP). Components of current EM field of UP 


$$
H_{\phi}=\frac{J_{0}}{2 \pi r} \exp (i \omega t-i \gamma z) \text {, when }\left|\nu_{c} r\right|<<1,
$$

which gives ground to simulate UP as linear power lines $[6 ; 8]$.

The basis of TUMM is EM field theory for cylindrical structures. Object complexity leads to cumbersome solutions, so it is methodologic expedient to consider simplified models (equivalent circuits, linear currents). Characteristics of magnetic field spatial distribution is the basis for choice of measured parameters (input values) and construction of algorithms, non-contact CM systems and UP coordinates. TUMM makes available to efficiently explore electromagnetic fenomena, connected to UP corrosion state, facilitate detection and analysis of EM field informative signs and development of methods and CP inspection systems, is theoretical basis of EM IMS [6; 8].

Using TUMM spatial characteristics of low-frequency current field (Figure 4-5) along the route and UP leakage current $[6 ; 8 ; 9]$ is analyzed. Relationships between geometrical and electric UP parameters and its EM field characteristics are explored, informative sings and range of measured values are detected. Analysis of pipeline phenomenological circuit is performed, simplified equivalent circuits for partial cases of direct and alternating current is build, method for estimation of area of UP insulation damaged is proposed [6]. Dependencies of distribution characteristics and pipeline EM field spreading on its parameters, insulation state and conductivity of medium is calculated. Required parameters of measuring transformers and devices $[6 ; 8]$ are determined; algorithms for input signal processing and determination of measured values (currents, voltages, resistances) as informative signs for estimation of UP CP state are created [10].

\section{Non-contact UP CM}

There is three method to measure current along pipeline without rupture of power line: 1 - due to voltage on pipeline region with known resistance using Ohm's law; 2 - due to magnetic flux in closed closed loop covering power line using Ampère's circuital law; 3 - due to characteristics of current magnetic field spatial distribution using Biot-Savart law. First method requires contact with pipeline metal. Second method is non-contact and integral, but requires circular access to pipeline. Third method is differential and can be apply to our problem. 
Theoretical basics of differential method of non-contact $\mathrm{CM}$ as a basis for systems of input transformers of non-contact UP CM design is developed $[4 ; 6 ; 8 ; 10]$. Classes of gradient (radial) and parallax (azimuthal) methods are distinguished. Their informative, metrological and technological properties are analyzed and compared. New methods of noncontact CM with azimuthal and radial orientation of the base of observation points, with arbitrary placement of base in transverse to power line plane (with component and modul primary transformers) (Figure 4) are proposed. Also cyclic method with moving of observation points on a circular trajectory is proposed.
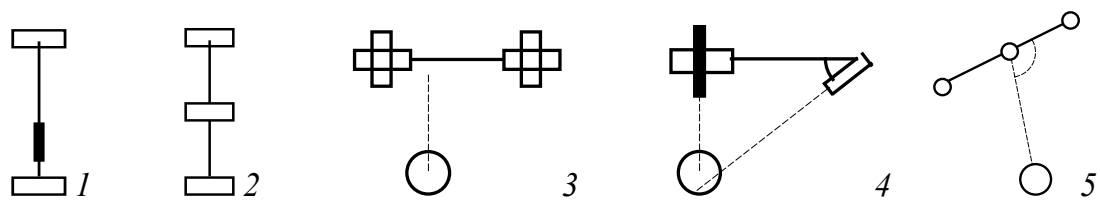

Figure 4. Method of non-contact CM:

1, 2 - radial; 4 - azimuthal; 3, 5 - invariant; 2 - difference

New methods and devices for non-contact CM are proposed. For express UP inspection in order to prevent damages of pipelines apparatus BIT-KVP $[4 ; 6 ; 8]$ is developed. This device provides pipeline and power lines placement, orientation and depth of occurrence determination and CM with relief error correction without access to pipeline and ground. It is also additionaly equipped with voltmeter for traditional contact CM and electronic memory. Results of measurements are translated to personal computer for automatic processing and documentation via interface using special program.

Conducted in PMI of the NASU with Lvivtransgaz theoretical and experimental researches of EM characteristics on UP routes and comparative methods and tools for diagnostic inspection show that for express testing of CP UP non-contact differential measurements of variable component of rectified pulsating current of SCP are most suitable. This current contains information both about the electromechanical (active) protection current distribution and about insulation state (passive protection) on different UP regions [10-12]. Characteristics of natural, man-made and technological field source on routes as interfering and SCP currents as informative are analyzed. 
Influence of current leakage (from UP to the ground) on magnetic field behavior (Figure 5) and on results of non-contact measurements of depth $h$ of UP occurrence and current using gradient $J_{g}$ and parallax $J_{p}$ methods are analyzed. It is showed theoretically and was confirmed experimentally, that local current leakage creates anomalies in distribution of measurements $h(l)$ and $J(l)$ along UP. It makes available to use this measurements for differentiation of local insulation damages, which are distant from each other at a distance not less than depth of UP occurrence [6;8].

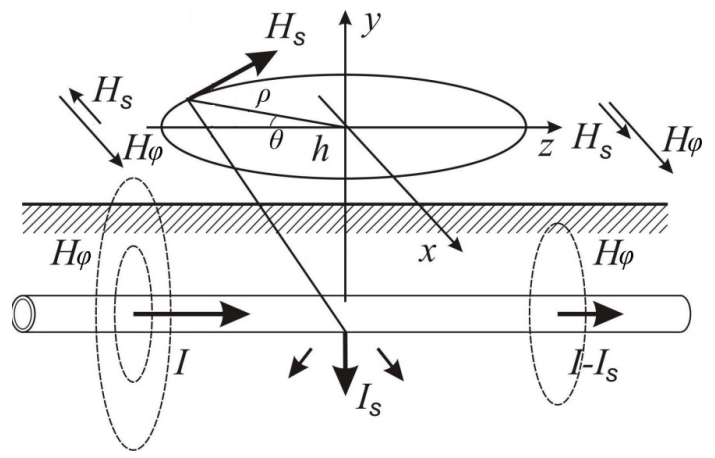

\section{Figure 5. Magnetic fields components: $H \varphi$ of transit current $I$ and $H s$ of current $I s$, that leaks through damage in UP insulation}

\section{Technology of non-contact UP inspection}

Methods for measurement of SCP current distribution between different threads and shoulders of pipelines (Figure 6, 7) and determination of UP CP current density using non-contact $\mathrm{CM}$ and harmonic coefficient for arbitrary point in SCP action area are proposed. Methods for detection of transient resistance "pipe-to-ground" on different UP regions and its components (ground, insulation and polarization resistances) are developed [12]. New method for detection of metal surface polarization potential in conductive medium with the exception of ohmic component by measurements of constant and variable voltages is proposed.

Concept is proposed and new information technology for CP UP state inspection based on non-contact $\mathrm{CM}$ for express detection of most probable corrosion places with further use of improved compact electrometry in 
places of abnormally large SCP current consumption for estimation of insulation and electrochemical protection state to prevent UP damages is developed.

Created tools make express integral (Figure 6) and differential (Figure 7,8 ) inspections and control $[4 ; 6 ; 10 ; 11]$ of CP parameters possible. Also local $[4 ; 6 ; 12]$ diagnostics inspections of underground metallic constructions corrosion state are available. Results of measurements on route automatically are stored in memory of device and translated to personal computer for automatic processing and documentation via interface using special program $[4 ; 6 ; 13]$.

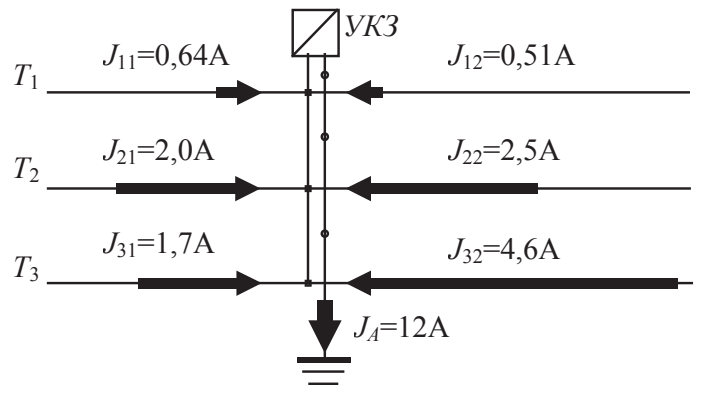

Figure 6. SCP current distribution by shoulders of three UP

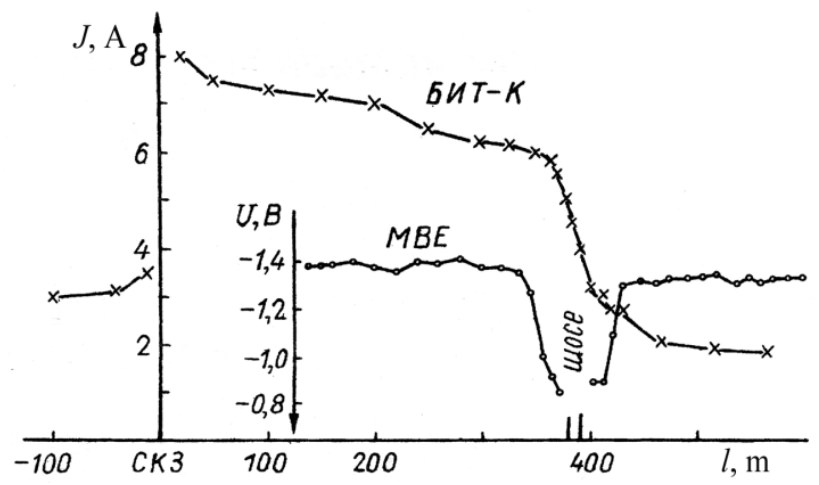

Figure 7. Non-contact CM SCP and contact measurement of UP potential 
Field tests and operation confirm, that non-contact CM method and EM IMS created using it can obtain new information about UP corrosion state, in particular: SCP current distribution, integral (Figure 6) and differential (Figure 7) quantitative estimations of insulation state on different regions without opening the pipeline. Functional possibilities of non-contact $\mathrm{CM}$ method, created devices and developed technology are confirmed by comparative testing and examples of use in express inspection of arterial oil and gas pipelines $[4 ; 6 ; 10-13]$.

Created devices of type ORT or non-compact CM are put into operation; they are used in Ukraine, Russia, Kazakhstan for diagnostics inspections of arterial pipelines, pipeline networks in human settlements and on territory of enterprises, other conductive communications.

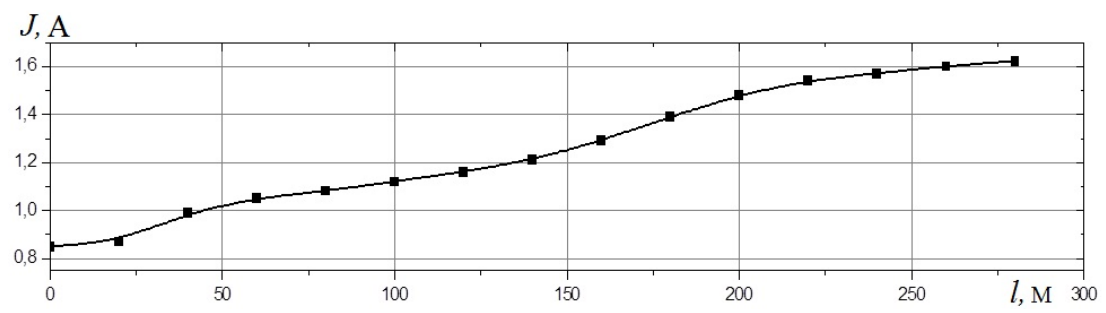

Figure $8 a$. Current $J(l)$ distribution along arterial pipeline using data obtained by non-compact device BMC-K

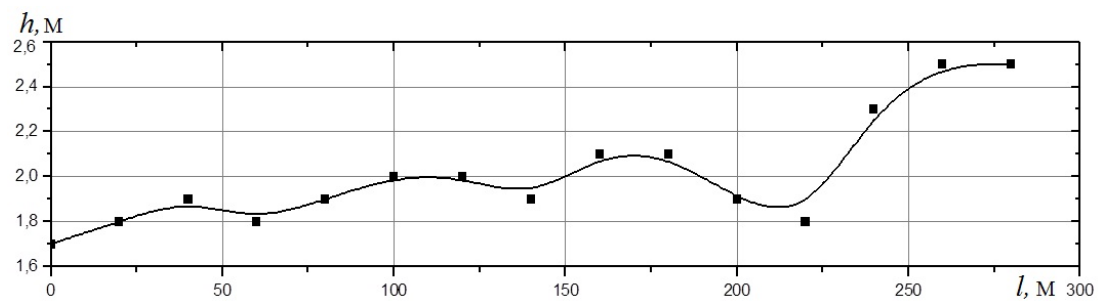

Figure $8 b$. Distribution of depth of occurrence measurements along arterial pipeline using data obtained by BMC-K

5.1. Detection of unsatisfactory UP insulation using current measurements. Using non-contact methods we can measure value of current Jn in UP points $n=0,1,2 \ldots$, distance between which are $\Delta l_{n}$. We can calculate current losses on each interval $\Delta l_{n}$ of the pipeline 


$$
\Delta J_{n}=J_{n}-J_{n-1}, \mathrm{~A}
$$

Relative current losses on unit of pipeline length (relative linear current leakage density) we can obtain using formulae

$$
\delta J_{n}=\frac{\Delta J_{n}}{J_{n c} \cdot \Delta l_{n}} \cdot 100, \% / \mathrm{M},
$$

where $J_{n c}=\left(J_{n}+J_{n-1}\right) / 2$ is mean current in pipeline on nth interval.

Relative linear current losses density $\delta J_{n}$ equals attenuation of signal along UP. It is proportional to conductivity of insulation and is UP insulation damage indicator: the largest values $\delta J_{n}$ indicate places with the largest conductivity "pipe-to-ground", i.e, the least resistance of insulation.

If relative current losses in pipeline on certain interval is bigger $\delta J_{n}>\delta J_{\kappa p}$ then critical value, which is detected by attenuation of EM wave in ground and obtained by formulae $[4 ; 6]$

$$
\delta J_{k p}=0,2 \sqrt{f / \rho_{g}}, \% / \mathrm{m},
$$

where $f$ is current frequency $(\mathrm{Hz}), \rho_{g}$ is ground resistivity $(\mathrm{Ohm} \bullet \mathrm{m})$, then it can be affirmed $[4 ; 6]$, that insulation on this nth interval of given UP region is unsatisfactory. Distribution of relative current losses $\delta J$, calculated using formulae (4), is showed on Figure 9. Overrun of its critical value $\delta J_{\kappa p}$ indicate places of unsatisfactory insulation.

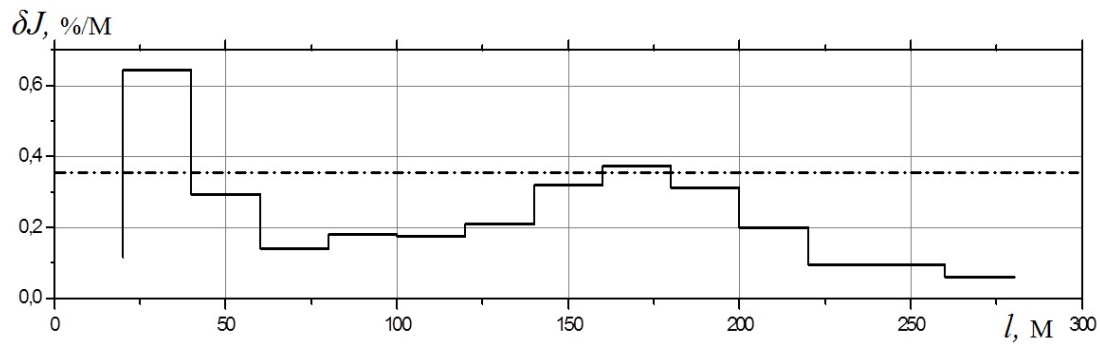

Figure 9. Distribution of relative current losses $\delta J$ along arterial pipeline; critical relative loss $\delta J_{k p}=\mathbf{0 , 3 6} \% / \mathrm{m}$

5.2. Transient resistance "pipe-to-ground" on unit of pipeline surface for each interval of $n$ UP region $[4 ; 6 ; 8 ; 10]$ is calculated by formulae

$$
R_{p g}(n)=\frac{R_{b} \pi D}{\left|\delta J_{n}\right|}, \Omega \mathrm{m}^{2},
$$


where $R_{b}=\frac{V_{p g}}{J_{p}}$ is input resistance of pipeline; $V_{p g}$ is voltage pipe-to-ground and $J_{p}$ is current in pipeline measured on the beginning of the section (from the current source). This values of transient resistance make possible to compare protection cover state (considering ground) on different intervals (sections) of UP.

\subsection{Detection of cathodic protection current density distribution.} Using measured current losses (3) we can obtain current leakage density on unit of UP surface for given section by formulae

$$
j_{n}=\Delta J_{n} / s_{n},
$$

where $\mathrm{s}$ is area of controlled UP section (for one-threaded pipeline $s=$ $\pi D \cdot \Delta l ; \Delta l$ is section length). During inspection of ramifications of pipelines, or their technological connections, currents of all pipeline branches and connected to them power lines are measured and total area of underground metallic surface on controlled section is calculated.

Orientation and value of constant component of rectified SCP current during inductive non-contact measurements can be obtain by comparing harmonics of current magnetic field using proposed methods $[4 ; 6 ; 10]$. Besides, current constant component density, which leaks to the pipeline from ground on given sections, can be calculated by current variable component density (7) with use of harmonic coefficient

$$
i_{n}=j_{n} / k_{n} \text {. }
$$

Harmonic coefficient can be determined by ratio of variable $\mathrm{Vg}$ and constant Ug voltages in ground across UP route $k_{n}=V_{g n} / U_{g n}$.

For cathodic polarization current density in places of insulation damage with area $s_{d n}$ on $n$th UP section, considering (3), (7) and (8), we have:

$$
i_{p n}=\Delta J_{n} / s_{d n} k_{n} \text {. }
$$

\subsection{Determination of UP transient resistance components}

Transient resistance (TR) is important generalized characteristic of UP protection covers. Values of $R_{p g}$ components is needed during planning and control of CP and diagnostics of its technical state. It is known, that pipelines with same insulation, which is embedded in soil with different resistivity have different TR $[4 ; 12]$. For example, TR in solonchak is three times smaller then in mudrock (for equally insulated pipeline sections). Soil, that surrounds pipeline (environment), is important part of protection 
covers. Thus concept TR pipeline-far ground $R_{p g}$ and TR of insulation $R i-$ and other components must be distinguished. It must be taken into account during measurements, TR and its components determination and estimation of UP protection cover estimation.

Known methods of UP TR and its components measurements are labor consuming and sometimes inadequate to real conditions of metallic constructions operation $[1 ; 2 ; 8]$. Disadvantages of traditional contact methods of integral TR estimation are absence of information about actual condition of protection covers on different sections of control region and big errors associated with difficulties of precision determination of control region length, selective measurements of potential "pipe-to-ground" difference on measuring points and inadequate approach about uniform distribution of SCP current along all action area. This method weakly respond to single local decrease or increase of current between pipeline and environment, which can lead to wrong results and conclusions.

This disadvantages of contact methods of UP diagnostic inspection . Examples of practical UP insulation control with determination of TR and its components distribution along UP using measurements of BMC and VPP during arterial gas pipelines inspection given in the works $[4 ; 6 ; 10-13]$ and publications cited by them. In this section we describe methods of TR determination methods based on UP non-contact CM.

Total UP TR (TR "pipe-to-far ground") can be obtained by formulae (6)

Soil resistivity on UP route $\rho_{g}$, as main indicator of corrosion aggressiveness of environment, traditionally is measured by method of four electrodes on the side of the route (to exclude pipeline influence) [1-3]. But during pipeline trenching soil characteristics will be violated and can significantly differ from characteristics of the soil on the side of the route.

Unlike this method, non-contact $\mathrm{CM}$ can determine resistance, that directly surrounds the pipeline, located on given section at depth $h$ [4]. We use measurements of current leakage $\Delta J_{n}(3)$ and measurements of voltage $V_{x n}$ between two electrodes on ground surface at distance $x$ and formulae

$$
\rho_{g n}=2 \pi l_{n} V_{x n} / \Delta J_{n} \ln \left(1+x^{2} / h\right), \mathrm{Ohm} \cdot \mathrm{m}
$$

Advantages of this method, except control of pipeline surrounding soil, are possibility of $\rho g$ determination using regular non-contact measurements 
of current $J_{n}$ and UP depth of occurrence hn and contact measurement of electric voltage $V_{x n}=V_{g g}$, which are performed directly on the route during UP inspection. So, using this measurements during their processing (without installation of four electrodes and additional measurements) $\rho_{g}$ distribution along UP.

Insulation resistance (insulated layer with possible pores and defects) on UP section in alternating low-frequency current can be considering as active (its reactive component can be neglected). It makes sense when with increasing of current frequency polarization resistance "metal-toenvironment" no longer manifests itself as well as capacitive (reactive) component of insulation (surrounded by soil) conductivity comparing to ohmic (active) component of conductivity [6]. Then resistance of this real (saturated with moisture, with pores and defects) UP insulated layer in operation can be obtain by formulae

$$
R_{\text {in }}=V_{\text {in }} / j_{n}, \Omega \cdot \mathrm{m}^{2},
$$

where current leakage density $j_{n}$ on given section can be determined using formula (7). Alternating voltage on insulated layer $V_{\text {in }}$ can be calculated by measurements of alternating voltages $V_{T g}$ between pipeline metal and reference electrode, located on ground above UP, and $V_{g g}$ on ground between two electrodes, spaced apart at distance $\mathrm{x}$. Using solution of electrodynamic boundary problem in $[6 ; 8]$ was showed that if distance $x$ choose considering depth of occurrence and UP diameter, than measured voltage on ground above UP $V_{g g}=V_{x}$ equals voltage drop VG in ground above pipeline $V_{g g}=V_{G}$. Then for determination of insulation resistance we obtain voltage on insulated layer:

$$
V_{i n}=V_{T g}-V_{g g} .
$$

Example of TR "pipe-to-ground" $R_{p g}$ and UP insulation TR $R_{i}$ due to non-contact CM on different section of UP is showed on Figure 10.

"Metal-to-ground" resistance in direct current on UP section, similar to $(11)$ is determined by ratio

$$
R_{\text {Tgin }}=U_{\text {in }} / i_{n}, \mathrm{OM} \cdot \mathrm{M}^{2}, \mathrm{Ohm} \cdot \mathrm{m}^{2}
$$

where, unlike (11), polarization potential $U_{\text {pol }}$ must be considered

$$
U_{i n}=U_{T g}-U_{g g}-U_{p o l}
$$




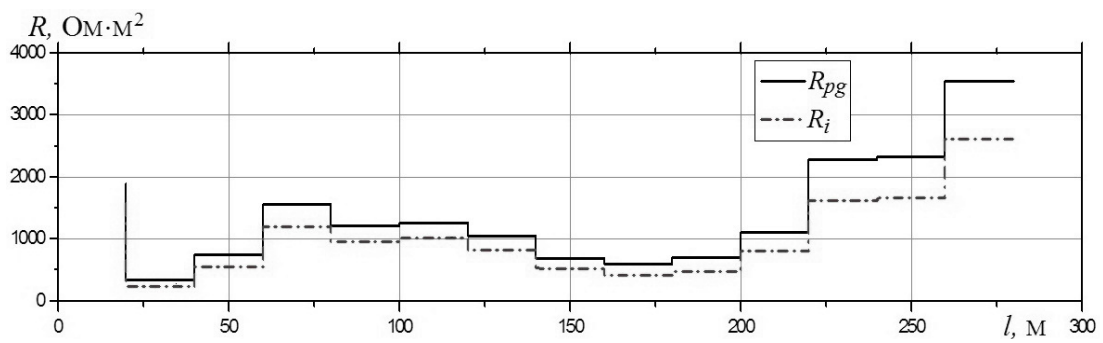

Figure 10. UP TR "pipe-to-ground" $R_{p g}$ and UP insulation TR $R_{i}$ distribution along arterial pipeline

and direct current density in can be determined using results of noncontact CM and harmonc coefficient by formuals (7), (8).

The obtained value (13) of "metal-to-gound" TR in direct current contains both insulation resistance $R_{\text {in }}$ and polarization resistance $R_{p o l}$ on given section. It can be used to calculate UP cathodic polarization.

Polarization resistance of unit of surface for given UP section by obtained TR "metal-to-ground" and insulation resistance components can be determined as:

$$
R_{\text {pol }}=R_{\text {Tgin }}-R_{\text {in }} \text {. }
$$

Taking into account similar to (9) area of insulation damages we obtain polarization resistance in places, where UP metal have contact with soil.

The obtained values of UP polarization resistance can be used for estimation of residual corrosion speed $[1 ; 4 ; 10-12]$ in places of insulation damages in real conditions of underground metallic constructions occurrence.

\section{Non-contact $\mathrm{CM}$ processing under influence of interference}

Current in UP monotonically decreases by exponential law with distance from current source. Inhomogeneity along the route lead to changes of attenuation current coefficient. Places of UP insulation damages can be determined by these changes as well TR can be determined by values of current losses. But monotony of UP current changes with distance from source in its action area converges [11].

However in practice of testing measurement errors can lead to violation of monotony of current changes along the route. Then direct application of 


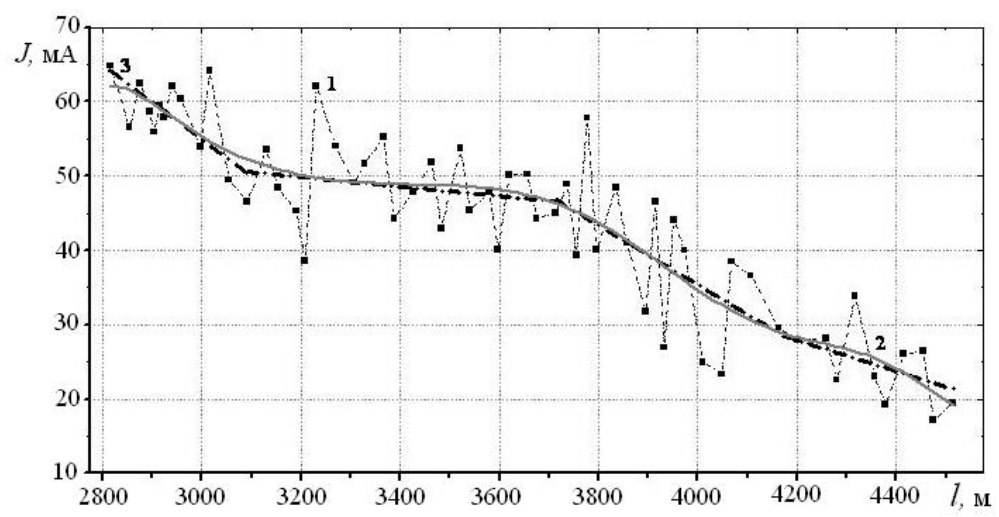

Figure 11. Distribution along the section of the main pipeline current measurements $J(l)$ :

1 - measurement results; 2 - approximation of measurements by a polynomial; 3 - approximation by linear dependences

formulas (3), (4) and (7) for determination of current losses can lead to the wrong results. Violation of monotony of current measurements results is true sign of the presence of signifycant errors, which must be excluded or corrected during data processing (Figure 11).

Reasons of non-contact CM error can be high level of interference (comparing to useful signal), inhomogeneity of the route, violation of normal equipment operation and human factor.

For correcting of array of measurements firsly single cases (measurement error) are excluded. In case of little changes of the measurement results for their correcting smoothing can be used [11]. During measurements in places of powerful interference, violations of monotony of current measurements are much larger and require more detailed analysis. Array of measurements can be divided on sections, inside which mean values of measurements lied on line; it is piecewise linear approximation. Approximation using method of least square can be use too (Figure 11).

Only when changes of current measurements (along the UP route in source action area), algorithms, described above, can be used properly. 


\section{Conclusions}

Important steel UPs are protected from corrosion using insulation and cathodic polarization. For control of their state traditionally contact electrometric methods are used. Disadvantages of known UP control methods are labor consuming and insufficient informativeness.

Efficiency of the UP inspections is significantly increased by use of noncontact methods, in particular, non-contact current measurement (NCM). For their broad use appropriate measured data processing tools are required.

In PMI of the NASU triune mathematical model of UP electromagnetic field are proposed. This model is based on solution of electrodynamic boundary value problem, theory of electric circuits with distributed parameters and calculations of spreading current electric and magnetic fields in conductive medium. Method of non-contact current measurement is developed. New BMC-type devices for control of corrosion protection state of the UP in operation are created.

New technology of integral, differential and local inspections and estimation of UP corrosion protection parameters are developed. New methods of efficient detection of places with unsatisfactory UP insulation, determination of cathodic protection current density along the route and transient resistance "pipe-to-ground" and its components (insulation, soil and polarization resistance) are proposed.

Efficiency of created method and equipment for non-contact inspection are tested by comparing with results of traditional contact electrometry, performed during diagnostic surveys of UP in operation. Developed method and NCM tools significantly increase efficiency of inspection, informativeness and reliability of results of UP corrosion protection control.

\section{References:}

1. Strizhevskii, I. V., \& Zinevich, A. M. (1981). Zashchita metallicheskikh sooruzheniy ot podzemnoi korrozii. Spravochnik. Moskva: Nedra, 293 p. (in Russian)

2. Baeckmann, V., \& Schvenk, V. (1984). Katodnaya zaschita ot korrozii / Per. s nem. Pod red. I. V. Strizhevskogo. Moskva: Metallurgiya, 495 p. . (in Russian)

3. Uhlig, H. H., \& Revie, R. W. (1989). Corrosion and corrosion control. New York: Wiley Publ, 456 p.

4. Dzhala, R. M. (2009). Bases of inspection and control of corrosion state of underground pipelines. Fracture mechanics and strength of materials: Reference manual / Ed.-in-chief V.V. Panasyuk. Vol. 11. Strength and durability of oil and gas pipelines and storage tanks / Ed. H.M. Nykyforchyn. Lviv: Spolom, pp. 143-184. (in Ukrainian) 
5. Cosham, A., \& Hopkins, P. (2004). "The assessment of corrosion in pipelines - guidance in the pipeline," Pipeline Pigging and Integrity Management Conference. 17-18th May 2004, Amsterdam, The Netherlands, pp. 1-31.

6. Dzhala, R. M. (ed.) (2018). Technical diagnostics of materials and structures: Reference manual / Ed.-in-chief Z. T. Nazarchuk. Vol. 4: Electrophysical methods for nondestructive testing of defects in structural elements / Dzhala R. M. (ed.), Dzhala V. R., Ivasiv I. B., Rybachuk V. G., Uchanin V. M. Lviv: Prostir-M., 356 p. (in Ukrainian)

7. Barnes, P. R. (1991). The magnetically inferred current attenuation method for pipeline coating evaluation // 2-nd International conference "Pipeline inspection". Moscow: MSIA "Spectrum", pp. 538-545.

8. Dzhala R. M. (2001). Electromagnetic survey and control of corrosion of pipelines // Mechanics of fracture and strength of materials: References. manual / Under edit V. V. Panasyuk. T.5: Non-destructive testing and technical diagnostics / Ed. Z. T. Nazarchuk. Lviv: G. V. Karpenko Institute of Physics and Mathematics of the National Academy of Sciences of Ukraine. Part. 5, pp. 263-330. (in Ukrainian)

9. L. Dikmarova, R. Dzhala, V. Nichoga, P. Dub (2001). External Electromagnetic Field of Underground Pipeline in Problems of Corrozion Protection // Proc. 5th International Conference on Applied Electromagnetics. Nis (Yugoslavia), pp. 41-44.

10. R. M. Dzhala, B. Ya. Verbenets', M. I. Mel'nyk, A. B. Mytsyk, R.S. Savula, O. M. Semenyuk (2017). New methods for the corrosion monitoring of underground pipelines according to the measurement of currents and potentials. Materials Science. Vol. 52, № 5. March 2017, pp. 732-741.

11. R. Dzhala, B. Verbenets', M. Mel'nyk, R. Savula, O. Semenyuk (2014). Control of corrosion protection of main pipelines with contactless measurement currents in the disturbances. PCMM. Special Issue № 10: Problems of Corrosion Protection of Materials. Vol. 2, pp. 539-544. (in Ukrainian)

12. R. Dzhala, V. Dzhala, R. Savula, O. Senyuk, B. Verbenets' (2019). Determination of components of transient resistance of underground pipeline. Elsevier Procedia Structural Integrity, Vol. 16, pp. 218-222.

13. R. Dzhala, V. Dzhala, B. Horon, O. Senyuk, B. Verbenets (2019). Information Technology of Surveys and Diagnostics of Underground Pipelines XIth International Scientific and Practical Conference on Electronics and Information Technologies (ELIT). Lviv: Ivan Franko National University, IEEE Ukraine section. September 16-18, 2019. IEEE Electronic Publication eCF Paper Id: 206299. Proceedings, pp. 214-217. 